\title{
Traversable wormholes with arbitrarily small energy condition violations
}

\author{
Matt Visser* \\ School of Mathematical and Computing Sciences, Victoria University of Wellington, New Zealand \\ Sayan Kar \\ Department of Physics and Centre for Theoretical Studies \\ Indian Institute of Technology, Kharagpur 721 302, WB, India \\ Naresh Dadhich $\ddagger$ \\ Inter-University Centre for Astronomy and Astrophysics \\ Post Bag 4, Ganeshkhind, Pune 411 00\%, India
}

(Dated: 1 January 2003; Revised 28 March 2003; LATEX-ed February 7, 2008)

\begin{abstract}
Traversable wormholes necessarily require violations of the averaged null energy condition; this being the definition of "exotic matter". However, the theorems which guarantee the energy condition violation are remarkably silent when it comes to making quantitative statements regarding the "total amount" of energy condition violating matter in the spacetime. We develop a suitable measure for quantifying this notion, and demonstrate the existence of spacetime geometries containing traversable wormholes that are supported by arbitrarily small quantities of "exotic matter".
\end{abstract}

PACS numbers: 04.70.Dy, 04.62.+v,11.10.Kk

Keywords: Lorentzian wormholes, exotic matter

Introduction: We know that traversable wormholes require "exotic matter", that is, violations of the averaged null energy condition [ANEC] 1, 2, 3, 4]. But, do we need "large" amounts of ANEC violating matter or are "small" amounts sufficient to do the job? This question is particularly interesting in view of the fact that quantum effects are known to induce some energy condition violations [5]. Furthermore numerical evidence suggests that quantum effects may be sufficient to support a wormhole throat 6]. On the other hand, there are significant limitations (the Ford-Roman "quantum inequalities" and their variants) on the "size" and "distribution" of quantum-induced energy condition violations [7].

To set the stage, consider the four great results of global analysis in classical GR - the area increase, positive mass, singularity, and topological censorship theorems. Some of these classical results seem to survive the introduction of quantum physics, others do not.

In the case of the area increase theorem (see, e.g., [8]) we know that quantum effects, though extremely tiny, violate the ANEC (and other energy conditions) and are a necessary pre-condition for Hawking radiation 9]. The effect though tiny is secular, so it accumulates and completely reverses the conclusion of the classical area increase theorem once quantum effects are brought into account.

In contrast, for the positive mass theorem [10] the sit-

\footnotetext{
*Electronic address: matt.visser@vuw.ac.nz

URL: http://www.mcs.vuw.ac.nz/ visser

${ }^{\dagger}$ Electronic address: sayan@cts.iitkgp.ernet.in

¥Electronic address: nkd@iucaa.ernet.in
}

uation is rather different. For a finite spherically symmetric static distribution we know (using curvature coordinates) that

$$
m_{\infty}=m_{0}+\int_{\max \left\{0,2 m_{0}\right\}}^{\infty} 4 \pi r^{2} \rho(r) \mathrm{d} r .
$$

So if the asymptotic mass is to be "large" and negative, then similarly either the central "bare" mass $m_{0}$ or the integrated density must be large and negative. You cannot get large negative asymptotic mass from infinitesimal weak energy condition [WEC] violations.

The situation with respect to the singularity theorems (see, e.g., [8]) is intermediate. The various ways currently known of violating the energy conditions [11] lead to "technical" violation of the singularity theorems - the hypotheses of the singularity theorems (as currently formulated) are not satisfied by empirical reality. Whether it is possible to avoid singularities armed only with arbitrarily small quantities of ANEC violating matter is not presently known.

With regard to topological censorship, the key question is this: Is the existence of traversable wormholes more akin to the situation with the area increase theorem (infinitesimal ANEC violations reverse the conclusion of the theorem) or is it more akin to the situation with regard to positive mass (large WEC violations needed for a large negative mass)? We shall answer this question by explicit example — we work with static spherically symmetric spacetimes and develop a suitable quantitative measure of the "total amount" of exotic matter. We then display a particular class of spacetime geometries that contain a traversable wormhole (and thereby violate topological censorship), but which are supported by 
arbitrarily small quantities of exotic matter.

We conclude that the topological censorship theorem is more akin to the area increase theorem than to the positive mass theorem - small violations of the energy conditions are sufficient to evade the conclusions of the theorem.

Constructing the wormhole: Consider a static spherically symmetric spacetime and go to Schwarzschild coordinates (curvature coordinates) [1, 4]:

$\mathrm{d} s^{2}=-\exp [2 \phi(r)] \mathrm{d} t^{2}+\frac{\mathrm{d} r^{2}}{1-b(r) / r}+r^{2}\left(\mathrm{~d} \theta^{2}+\sin ^{2} \theta \mathrm{d} \varphi^{2}\right)$

Then, using the Einstein field equations, the components of the diagonal energy-momentum tensor in an orthonormal basis turn out to be (units $-G=c=1$ ) [1, 4] :

$$
\rho=\frac{1}{8 \pi} \frac{b^{\prime}}{r^{2}} \quad ; \quad p_{r}=\frac{1}{8 \pi}\left[-\frac{b}{r^{3}}+2\left\{1-\frac{b}{r}\right\} \frac{\phi^{\prime}}{r}\right]
$$

$p_{t}=\frac{1}{8 \pi}\left\{1-\frac{b}{r}\right\}\left[\phi^{\prime \prime}+\phi^{\prime}\left(\phi^{\prime}+\frac{1}{r}\right)\right]-\frac{1}{2}\left(\frac{b}{r}\right)^{\prime}\left(\phi^{\prime}+\frac{1}{r}\right)$

where $\rho, p_{r}$, and $p_{t}$ are the energy density, the radial and tangential pressures respectively. If the equation $b(r)=r$ has a nontrivial solution $r_{0}$, and $\exp \left[\phi\left(r_{0}\right)\right] \neq 0$, then we can cut the spacetime at $r=r_{0}$, and paste it onto a second copy of itself, with a $C^{2}$ geometry at $r=r_{0}$. The extrinsic curvature of the hypersurface $r=r_{0}$ ( $\eta$ is proper radial distance) is

$$
K_{a b} \propto \frac{\partial h_{a b}}{\partial \eta}=\frac{\partial h_{a b}}{\partial r} \frac{\partial r}{\partial \eta}=\frac{\partial h_{a b}}{\partial r} \sqrt{1-b(r) / r} \rightarrow 0 .
$$

As long as $\exp \left[\phi\left(r_{0}\right)\right] \neq 0$ you can go to an orthonormal basis and have $K_{\hat{a} \hat{b}}=0$. (Hatted indices denote components in an orthonormal basis.) The junction condition formalism 12] now guarantees the geometry is $C^{2}$ across the gluing hypersurface. (Under normal conditions the junction formalism yields a $C^{1}$ geometry, it is the vanishing of the extrinsic curvature at the junction that in this case makes the geometry $C^{2}$.)

The ANEC integral along a radial null geodesic is

$$
I=\oint\left[\rho+p_{r}\right] \exp [-2 \phi] \mathrm{d} \lambda=\oint\left[\rho+p_{r}\right] \exp [-\phi] \mathrm{d} \eta
$$

An integration by parts yields (see pp. 133-134 of [4])

$$
I=-\frac{1}{4 \pi} \oint \frac{1}{r^{2}} e^{-\phi} \sqrt{1-\frac{b}{r}} \mathrm{~d} r<0 .
$$

Unfortunately this is a line integral, with dimensions (mass)/(area), not a volume integral, and so gives no useful information regarding the "total amount" of energycondition violating matter.

The basic volume-integral theorem relates the asymptotic mass to the throat radius $r_{0}$ and the density by

$$
m_{\infty}=\frac{r_{0}}{2}+\int_{r_{0}}^{\infty} 4 \pi r^{2} \rho(r) \mathrm{d} r
$$

This is a simple generalization of the ordinary mass formula for relativistic stars to traversable wormholes. 13] Now

$$
\int \mathrm{d} V=\int 4 \pi r^{2} \mathrm{~d} r ; \quad \oint \mathrm{d} V=2 \int_{r_{0}}^{\infty} 4 \pi r^{2} \mathrm{~d} r .
$$

is a very natural integration measure in Schwarzschild coordinates. This is the measure that appears in the simplest formula for the total mass. Indeed, including both asymptotic regions, we have

$$
\oint \rho \mathrm{d} V=2 m_{\infty}-r_{0}
$$

We now develop our key volume-integral result, using this same " $r^{2} \mathrm{~d} r$ " measure. It is easy to check that

$$
\rho+p_{r}=\frac{1}{8 \pi r}\left\{1-\frac{b}{r}\right\}\left[\ln \left(\frac{\exp [2 \phi]}{1-b / r}\right)\right]^{\prime} .
$$

Then integrating by parts

$$
\begin{aligned}
\oint\left[\rho+p_{r}\right] & \mathrm{d} V=\left[(r-b) \ln \left(\frac{\exp [2 \phi]}{1-b / r}\right)\right]_{r_{0}}^{\infty} \\
- & \int_{r_{0}}^{\infty}\left(1-b^{\prime}\right)\left[\ln \left(\frac{\exp [2 \phi]}{1-b / r}\right)\right] \mathrm{d} r
\end{aligned}
$$

The boundary term at $r_{0}$ vanishes by our construction of the wormhole. The boundary term at infinity vanishes because of the assumed asymptotic behaviour. Then

$$
\oint\left[\rho+p_{r}\right] \mathrm{d} V=-\int_{r_{0}}^{\infty}\left(1-b^{\prime}\right)\left[\ln \left(\frac{\exp [2 \phi]}{1-b / r}\right)\right] \mathrm{d} r
$$

This volume-integral theorem provides information about the "total amount" of ANEC violating matter in the spacetime. [14] For the transverse pressure we have

$$
p_{t}=p_{r}+\frac{r}{2}\left\{p_{r}^{\prime}+\left(\rho+p_{r}\right) \phi^{\prime}\right\}
$$

but in the general case this does not lead to a particularly useful volume integral. We emphasise that it is $p_{r}$ that is guaranteed to be associated with ANEC violations, whereas inequalities associated with $p_{t}$ generically represent "normal" matter.

Specialization 1: Spatial Schwarzschild. Now consider the special case where the spatial metric is exactly Schwarzschild, that is $b(r) \rightarrow 2 m=r_{0}$. Then $\rho=0$ throughout the spacetime and we get the very simple result

$$
\oint p_{r} \mathrm{~d} V=-\int_{r_{0}}^{\infty} \ln \left[\frac{\exp [2 \phi]}{1-2 m / r}\right] \mathrm{d} r
$$

Thus the total ANEC violating component of the stressenergy is finite and bounded. Suppose in particular that we have a wormhole whose field only deviates from 
Schwarzschild in the region from the throat out to radius $a$. Then we can further simplify the above to

$$
\oint p_{r} \mathrm{~d} V=-\int_{r_{0}}^{a} \ln \left[\frac{\exp [2 \phi]}{1-2 m / r}\right] \mathrm{d} r .
$$

Under this same restriction the ANEC integral satisfies

$$
I<-\frac{2}{4 \pi} \int_{a}^{\infty} \frac{1}{r^{2}} \mathrm{~d} r=-\frac{1}{2 \pi a},
$$

and so is strictly bounded away from zero. Now

$$
\int_{r_{0}}^{a} \ln \left[\frac{\exp [2 \phi]}{1-2 m / r}\right] \mathrm{d} r<\int_{r_{0}}^{a} \ln \left[\frac{\exp \left[2 \phi_{\max }\right]}{1-2 m / r}\right] \mathrm{d} r .
$$

Evaluating this last integral

$$
\oint p_{r} \mathrm{~d} V>-(a-2 m) \ln \left[\frac{\exp \left[2 \phi_{\max }\right]}{1-2 m / a}\right]-2 m \ln \left(\frac{a}{2 m}\right) \text {. }
$$

This is useful because it is an explicit lower bound on the total amount of radial stress in terms of $\phi_{\max }$ and the size of the region of ANEC violating matter. Similarly

$$
\oint p_{r} \mathrm{~d} V<-(a-2 m) \ln \left[\frac{\exp \left[2 \phi_{\min }\right]}{1-2 m / a}\right]-2 m \ln \left(\frac{a}{2 m}\right) .
$$

This is now an upper bound in terms of $\phi_{\min }$ and the size of the region of ANEC violating matter. If we now choose geometries such that $\phi_{\max }$ and $\phi_{\min }$ are not excessively divergent, [no worse than $(a-2 m)^{-\delta}$ with $\delta<1$ ], we can take the limit $a \rightarrow 2 m$ to obtain

$$
\oint p_{r} \mathrm{~d} V \rightarrow 0
$$

That is: By considering a sequence of traversable wormholes with suitably chosen $a$ and $\phi(r)$ [and $b(r)=2 m$ ] we can construct traversable wormholes with arbitrarily small quantities of ANEC-violating matter. (With the ANEC line integral nevertheless remaining finite and negative.) Since this result is rather important, we now provide an even more explicit example.

\section{Specialization 2: Piecewise $R=0$ wormhole.}

We now consider a segment of $R=0$ wormhole (zero Ricci scalar) 15] truncated and embedded in a Schwarzschild geometry. For $r \in\left(r_{0}=2 m, a\right)$ take

$$
\exp [\phi(r)]=\epsilon+\lambda \sqrt{1-2 m / r},
$$

and for $r \in(a, \infty)$ take

$$
\exp [\phi(r)]=\sqrt{1-2 m / r} .
$$

Continuity implies

so that

$$
\epsilon+\lambda \sqrt{1-2 m / a}=\sqrt{1-2 m / a},
$$

$$
\lambda=1-\frac{\epsilon}{\sqrt{1-2 m / a}} .
$$

There is ANEC violating matter confined to the region $\left[r_{0}, a\right)$ and a thin shell of quasi-normal matter at $r=a$. 16] (Quasi-normal meaning it's not ANEC violating, or for that matter violating other energy conditions, namely the Weak Energy Condition (WEC) or the Strong Energy Condition (SEC). It does however violate the Dominant Energy Condition (DEC); see [4] for details on the different energy conditions.

Computing the extrinsic curvature at $r=a>2 m$ :

$$
K_{a b}=\frac{\partial h_{a b}}{\partial \eta}=\frac{\partial h_{a b}}{\partial r} \frac{\partial r}{\partial \eta}=\frac{\partial h_{a b}}{\partial r} \sqrt{1-2 m / a} .
$$

Then the only non-trivial component is

$$
K_{t t}^{+}=\sqrt{1-2 m / a} \frac{2 m}{a^{2}},
$$

while

$$
K_{t t}^{-}=\lambda \sqrt{1-2 m / a} \frac{2 m}{a^{2}} .
$$

The only non-zero component of the discontinuity is

$$
\left[K_{t t}\right]=(1-\lambda) \frac{2 m}{a^{2}} \sqrt{1-2 m / a}=\epsilon \frac{2 m}{a^{2}} .
$$

In an orthonormal frame

$$
\left[K_{\hat{t} \hat{t}}\right]=\frac{\epsilon 2 m / a^{2}}{1-2 m / a} .
$$

Since the extrinsic curvature is non-zero, the junction condition formalism implies that the metric is $C^{1}$ at $r=$ $a$. The only component of stress-energy that picks up a delta-function contribution is $p_{t}$. That is

$$
\begin{gathered}
p_{r}=-\frac{1}{8 \pi} \frac{2 m \epsilon}{r^{3}(\epsilon+\lambda \sqrt{1-2 m / r})} \Theta(a-r) ; \\
p_{t}=\frac{1}{8 \pi} \frac{m \epsilon}{r^{3}(\epsilon+\lambda \sqrt{1-2 m / r})} \Theta(a-r) \\
+\frac{1}{8 \pi} \frac{\epsilon 2 m / a^{2}}{1-2 m / a} \delta\left(\eta-\eta_{a}\right) .
\end{gathered}
$$

Note that at the throat $(r=2 m)$

$$
p_{r}=-\frac{1}{8 \pi} \frac{1}{(2 m)^{2}}=-\frac{p_{t}}{2},
$$

both of which are finite for all $a$ and $\epsilon$.

The volume integral is

$$
\oint p_{r} \mathrm{~d} V=-2 m \epsilon \int_{2 m}^{a} \frac{\mathrm{d} r}{r(\epsilon+\lambda \sqrt{1-2 m / r})},
$$

which evaluates (after a little work) to 


$$
\oint p_{r} \mathrm{~d} V=-2 m \epsilon \epsilon_{s}\left\{\frac{\epsilon \epsilon_{s} \ln \left[\epsilon^{2}\left(1-\epsilon_{s}^{2}\right) / \epsilon_{s}^{2}\right]+\left(\epsilon-\epsilon_{s}\right) \ln \left[\left(1-\epsilon_{s}\right) /\left(1+\epsilon_{s}\right)\right]}{\left(\epsilon-\epsilon_{s}\right)^{2}-\epsilon^{2} \epsilon_{s}^{2}}\right\} .
$$

Here we have introduced

$$
\epsilon_{s}=\sqrt{1-2 m / a} .
$$

Once $\oint p_{r} \mathrm{~d} V$ is known, $\oint p_{t} \mathrm{~d} V$ is trivial. It is easiest to consider

$$
\oint\left[p_{r}+2 p_{t}\right] \mathrm{d} V=\frac{2 \epsilon m}{\sqrt{1-2 m / a}}=2 \frac{\epsilon}{\epsilon_{s}} m .
$$

The point is that $\oint p_{r} \mathrm{~d} V$ can be made arbitrarily small by suitably choosing $\epsilon$ and $a$. For example, take $\epsilon / \epsilon_{s}$ fixed, and let $\epsilon \rightarrow 0$. Now in that case $\oint\left[p_{r}+2 p_{t}\right] \mathrm{d} V$ remains finite, but you could just as easily choose $\epsilon_{s}=$ $\sqrt{\epsilon} \rightarrow 0$, or even $\epsilon_{s}$ fixed and $\epsilon \rightarrow 0$, in which case both integrals tend to zero. Thus the volume integrals of both $p_{r}$ and $p_{t}$ can both be made arbitrarily small while the volume integral of $\rho$ is identically zero by construction. In this particular example the geometry is sufficiently simple that an integration in terms of the proper volume $\sqrt{g_{3}} \mathrm{~d}^{3} x$ can also be explicitly carried out. Qualitatively similar results are obtained.

We emphasise that the particular details of the geometry we have written down are nowhere near as important as the general principle that energy condition violations can be made arbitrarily small.

Conclusions: The specific examples presented in this Letter make essential use of spherical symmetry, and it is not yet clear to us how to usefully extend these ideas to more general situations - that is however not critical to the central point of this Letter; any explicit example of a traversable wormhole with infinitesimal ANEC violations will serve to illustrate the point we wish to make.

Let us now summarize the key result: ANEC violations are certainly needed to support traversable wormholes, but by appropriate choice of the wormhole geometry the total quantity of ANEC violating matter can be made infinitesimally small. Quantum physics is known to lead to small violations of the ANEC, and indeed quantuminduced ANEC violations are known to be an essential precondition for the violation of the area-increase theorem engendered by the existence of Hawking radiation. Thus topological censorship shares with the area increase theorem the fact that its conclusions can be radically altered by subtle quantum effects. (In contrast, generating macroscopic violations of the positive mass theorem requires macroscopic violations of the energy conditions.)
[1] M. S. Morris and K. S. Thorne, Am. J. Phys. 56 (1988) 395.

M. S. Morris, K. S. Thorne and U. Yurtsever, Phys. Rev. Lett. 61 (1988) 1446.

[2] J. L. Friedman, K. Schleich and D. M. Witt, Phys. Rev. Lett. 71 (1993) 1486 [Erratum-ibid. 75 (1995) 1872] arXiv:gr-qc/9305017. K. Schleich and D. M. Witt, arXiv:gr-qc/9903061 G. J. Galloway, K. Schleich, D. M. Witt and E. Woolgar, Phys. Rev. D 60, 104039 (1999) arXiv:gr-qc/9902061.

[3] D. Hochberg and M. Visser, Phys. Rev. Lett. 81 (1998) 746 arXiv:gr-qc/9802048; Phys. Rev. D 56 (1997) 4745 arXiv:gr-qc/9704082.

[4] M. Visser, Lorentzian wormholes: from Einstein to Hawking, AIP Press (1995).

[5] H. Epstein, V. Glaser, and A. Jaffe, Nuovo Cim. 36 (1965) 1016.

[6] D. Hochberg, A. Popov and S. V. Sushkov, Phys. Rev. Lett. 78 (1997) 2050 arXiv:gr-qc/9701064.

[7] L. H. Ford and T. A. Roman, Phys. Rev. D 51 (1995) 4277 arXiv:gr-qc/9410043.

A. Borde, L. H. Ford and T. A. Roman, Phys. Rev. D 65 (2002) 084002 arXiv:gr-qc/0109061.

L. H. Ford, A. D. Helfer and T. A. Roman, Phys. Rev. D 66 (2002) 124012 arXiv:gr-qc/0208045.

[8] S. W. Hawking and G. F. R. Ellis, The large scale struc- ture of spacetime, (Cambridge, England, 1973).

[9] N. D. Birrell and P. C. W. Davies, Quantum fields in curved space, (Cambridge, England, 1982). See esp. pp. $270 \mathrm{ff}$.

[10] R. Schon and S. T. Yau, Commun. Math. Phys. 65 (1979) 45; Commun. Math. Phys. 79 (1981) 231.

[11] C. Barcelo and M. Visser, Int. J. Mod. Phys. D 11 (2002) 1553 arXiv:gr-qc/0205066.

[12] W. Israel, Nuovo Cim. B 44 S10 (1966) 1 [Erratum-ibid. B 48 S10 (1967) 463]. K. Lanczos, Ann. Phys. (Liepzig) 74 (1924) 518-540. N. Sen, Ann. Phys. (Liepzig) 73 (1924) 365-396.

[13] In order to be certain that $m_{\infty}$ corresponds to the physical mass, there is a technical requirement on the asymptotic behaviour of $g_{t t} g_{r r} \rightarrow-1+O\left(1 / r^{2}\right)$.

[14] If one is dealing with a star instead of a wormhole one obtains a similar formula with the RHS of (13) being modified by an (i) overall factor of one half and (ii) the integration limits becoming 0 to $\infty$.

[15] N. Dadhich, S. Kar, S. Mukherji and M. Visser, Phys. Rev. D 65 (2002) 064004 arXiv:gr-qc/0109069.

[16] The thin shell is present only as a mathematical convenience - any smooth transition from the wormhole region to the Schwarzschild region would be physically acceptable, but explicit evaluation of the integrals would be more difficult. 